\title{
Influence of 2D and 3D Meshes in FE Computation of Eddy-Current Losses in Surface PMSMs
}

\author{
Yves Mollet, Johan Gyselinck \\ Bio, Electro And Mechanical Systems Dept. \\ ULB Brussels, Belgium \\ ymollet@ulb.ac.be,johan.gyselinck@ulb.ac.be
}

\author{
Ruth V. Sabariego \\ Dept. of Electrical Engineering \\ Campus EnergyVille, KU Leuven, Belgium \\ Email: ruth.sabariego@kuleuven.be
}

\begin{abstract}
This paper investigates the influence of mesh when computing eddy currents in magnets of a permanent-magnet synchronous machine excited with pulsating currents (such as pulse-width modulation). 3D computations with globally and locally varying mesh densities are performed and results are compared with a reference (fine-mesh) case. It turns out that a global increase of mesh density has the most important impact on the result accuracy. The accuracy of $2 \mathrm{D} \mathrm{FE}$ computations is also discussed for different stack lengths. It is shown that the use of a correction factor based on analytical approach can significantly increase accuracy for relatively short stack lengths.
\end{abstract}

\section{INTRODUCTION}

Rare-earth permanent-magnet synchronous machines (PMSMs) are used for various applications, since they benefit from high compactness, efficiency and power factor [1]-[3]. However, rare-earth materials have a relative high conductivity [3] and eddy currents can develop in the permanent magnets (PMs). As the fundamental component of the magnetic flux is stationary with respect to the rotor, eddy-current losses are generally negligible compared to the other loss components [1]: only spatial flux harmonics (related to slotting and winding distribution) and temporal ones (due to switching) generate losses [1], [4], [5]. Despite this aspect, the temperature rise may be important due to the relative thermal insulation of the magnets [1] and deteriorate their properties (coercive field and remanent flux), possibly leading to their demagnetization [2], [5]. Furthermore, the PM losses become even more significant in megawatt-range machines (e.g. wind turbines) or high-speed applications (e.g. electrical and hybrid vehicles) [2], [4], [6].

For those reasons an accurate estimation of the eddy-current losses is necessary for design purposes [6], but it is not easy [1]. Analytical approaches are widely used but may not be valid for all geometries (e.g. for interior PMs) [6]. Furthermore, often they do not take the reaction field from the eddy currents into account, leading to inaccurate results at high frequencies [2], [4]. This aspect is, however, considered in the method developed in [2], which computes the induced currents generated by all relevant spatial and temporal harmonics. Total losses are computed by superposition principle, which is shown to be sufficiently accurate in [5]. 3D effects are also modelled in [2] by the multiplication of the losses obtained with a 2D model with a correction factor depending on the axial length based on analytical approach. In [4], a "quasi-3D" analytical solution is also developed by imposing a flux density normal to the PM surface and a zero normal component of current density in the magnets.

FE approaches are also widely used for estimating eddy currents. 2D models without consideration of the reaction field (eddy-current losses being computed in the post-processing phase) remain the main design tools [6], even if the average current is non-zero (PMs considered as conductor bars with both ends short-circuited at infinity). More advanced methods subtract this average value from the results, but their accuracy is limited because they neglect skin effect [2]. 3D FE computations are more accurate, but also much more computationally expensive [2], [4]. A combined 2D axial and radial method is proposed in [6] to avoid 3D FE computations. The flux obtained by the radial solver is used as a source for the axial solver and the eddy currents are assumed to flow in radial slices.

Most FE techniques use (adaptive) time stepping, which may require a huge number of steps before reaching steadystate [7]. Harmonic FE simulation are scarcely used except in static magnetic devices (transformers) and induction machines. Compared to time stepping, the system of equations is solved in one shot, but the coupling of the considered frequencies makes the system matrix denser, leading to a more expensive resolution [7].

The experimental investigation of eddy-current losses in electrical machines is also non-trivial, due to their complex geometry. As a consequence, a simpler magnetic circuit is chosen in [3] to develop a test device to validate results obtained with a corresponding 3D FE model. An estimation of PM losses in a PMSM from measurements is proposed in [5] by subtracting from the total losses the estimated Joule, statoriron and rotor-iron losses, computed using either analytical equations or FE computations.

In this paper the influence of the mesh on the FE-computed eddy-current losses is investigated. The studied machine and the FE modelling and computation approach are described in the next sections. In the following section the simulation results and computation time are compared in 2D while globally or locally refining the mesh in order to find optimal mesh-refinement parameters. This optimised 2D mesh is then extruded and 3D computations are then run to determine the influence of the number of axial layers and their distribution 
TABLE I

MAIN DIMENSIONS AND CHARACTERISTICS OF THE INVESTIGATED PMSM

\begin{tabular}{|c||c|}
\hline Parameter & Value \\
\hline Number of pole pairs & 4 \\
Number of stator slots & 24 \\
Series turns per phase & 416 \\
Number of conductors per slot & 104 \\
Outer stator radius [mm] & 46 \\
Inner stator radius [mm] & 26 \\
Stack length [mm] & 40 \\
Air-gap width [mm] & 0.55 \\
PM thickness [mm] & 2.2 \\
PM/pole arc length ratio & 0.75 \\
PM conductivity [MS/m] & 0.67 \\
PM relative permeability & 1 \\
PM remanent induction [T] & 1 \\
Nominal phase current [A] & 4 \\
Phase resistance (DC) [ $\Omega$ ] & 0.707 \\
\hline
\end{tabular}

on the results. Based on that comparison, one of the 3D mesh is selected as a reference to evaluate the accuracy of $2 \mathrm{D}$ with respect to $3 \mathrm{D}$ computations in function of the axial length of the machine.

\section{INVESTIGATED PMSM}

The present paper focuses on the influence of temporal harmonics of the armature current (in a range of $50 \mathrm{~Hz}$ to $100 \mathrm{kHz}$ to essentially investigate switching components) on the eddy-current losses in the permanent magnets. As the rotation of the rotor corresponds to a much lower frequency, it does not influence significantly the eddy currents resulting from switching harmonics. Therefore, all harmonic FE simulations are performed for a fixed rotor position and sinusoidally pulsating flux (i.e. with a fixed direction with respect to the rotor) and with deactivated DC magnet flux, (unless different conditions are specified) to get the steady-state solution at each investigated frequency.

FE calculations are performed on a 8-pole 3-phase PMSM with 24 slots on the stator (i.e. one slot per pole and per phase). The main dimensions and parameters of the PMSM are listed in Table I. The stator slots of the original machine are skewed and the magnets are positioned on the rotor in a slightly asymmetric way, so that the spatial harmonics are reduced [7]. As those harmonics are not taken into account in the present work, the slots are here considered as straight and the rotor as symmetric. One sixteenth of the machine is therefore modelled (or one eighth for 2D simulations), i.e. only one pole over half of the axial length. In order to illustrate the normal working conditions of the machine, the flux lines and the axial component of the magnetic vector potential are presented in Figure 1 considering static case with both contributions of the magnets (acting on the direct or $d$ axis) and of the nominal current (acting on the $q$ axis, i.e. in quadrature with respect to the PM flux).

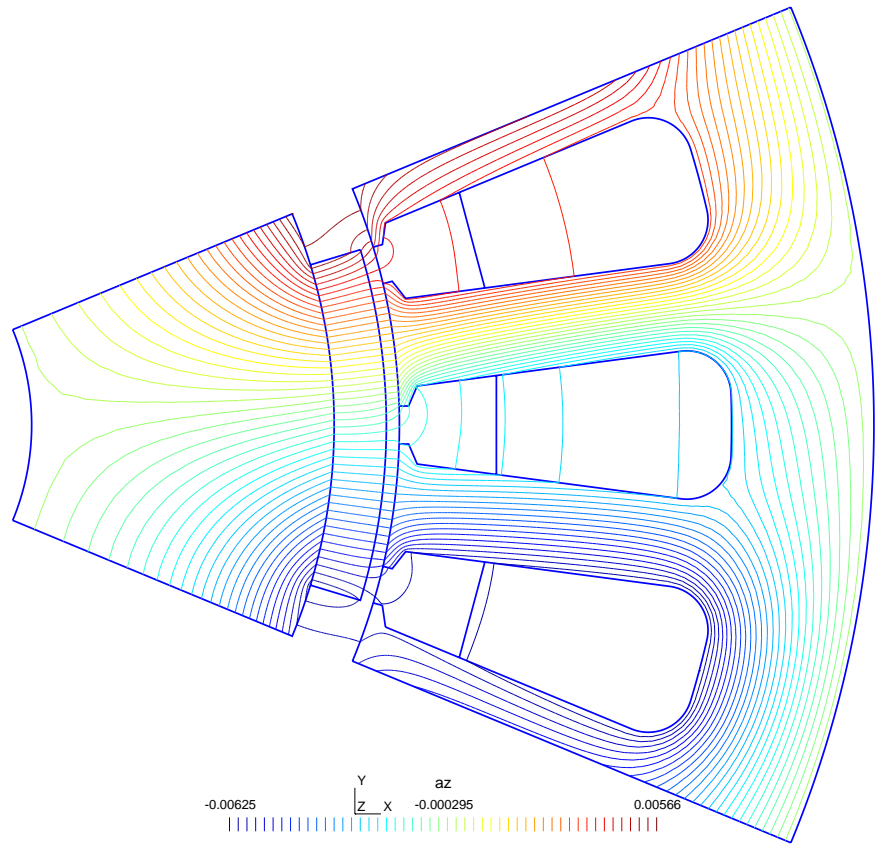

Fig. 1. Flux lines obtained when combining the action of the magnets and of the nominal current on the $q$ axis (static case). The $2 \mathrm{D}$ reference mesh described in the next section is used for the computation. The computed torque is $4.63 \mathrm{Nm}$.

The evolution of the characteristics of the machine with current frequency are also illustrated by the plots of the equivalent phase resistance and inductance of the winding and of the eddy-current losses in the PMs in a range from $50 \mathrm{~Hz}$ to $100 \mathrm{kHz}$ in Figures 2 and 3, respectively. These plots are generated by $2 \mathrm{D}$ and $3 \mathrm{D}$ FE calculations using the corresponding reference meshes described in the next section.

A significant increase of the resistance (equal to $0.707 \Omega$ at DC, i.e. value given by copper resistivity and geometrical considerations) is observed starting around $1 \mathrm{kHz}$. This can be explained by the rise of the eddy-current losses, which become predominant compared to the Joule losses in the stator winding at that frequency. Note that other phenomena can also influence the resistance, such as the skin and proximity effects inside the winding [1]; they are neglected as inductors are assumed to be stranded.

At higher frequencies (starting at about $10 \mathrm{kHz}$ ) the phase inductance starts decreasing. Referring to [4], $10 \mathrm{kHz}$ is the threshold at which the eddy currents are no more limited by the resistivity of the PM material (resistance-limited case) but start to shield the magnetic field generated by the winding [1], [5], preventing it from entering the magnets (inductance-limited case). As a consequence, the eddy currents concentrate on the periphery of the magnets. This effect is also visible in the PM losses (Figure 3), as they are no longer proportional to the square of the frequency. This last high-frequency range is also the one in which the segmentation of the magnets proposed in some papers to reduce losses start to be counter-productive [2], [4]. For the same reason PM segmentation in classical radial-flux machines only makes sense if the rotor back iron 


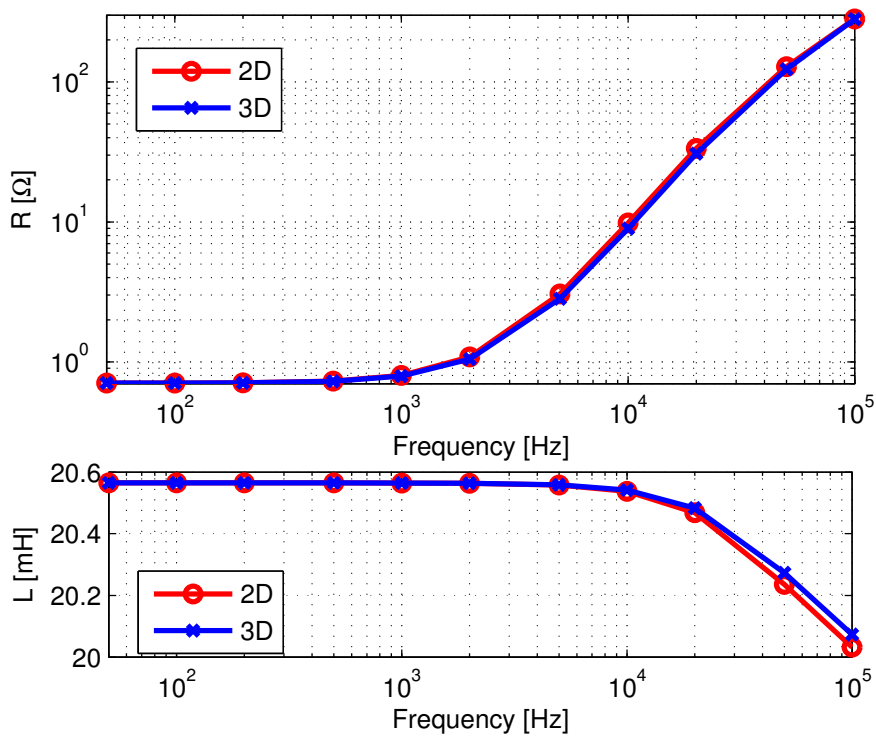

Fig. 2. Evolution of equivalent phase resistance $R$ and inductance $L$ of the winding with frequency for $1 \mathrm{~A} q$-axis current. The $2 \mathrm{D}$ and $3 \mathrm{D}$ reference meshes described in the next section are used for the computations.

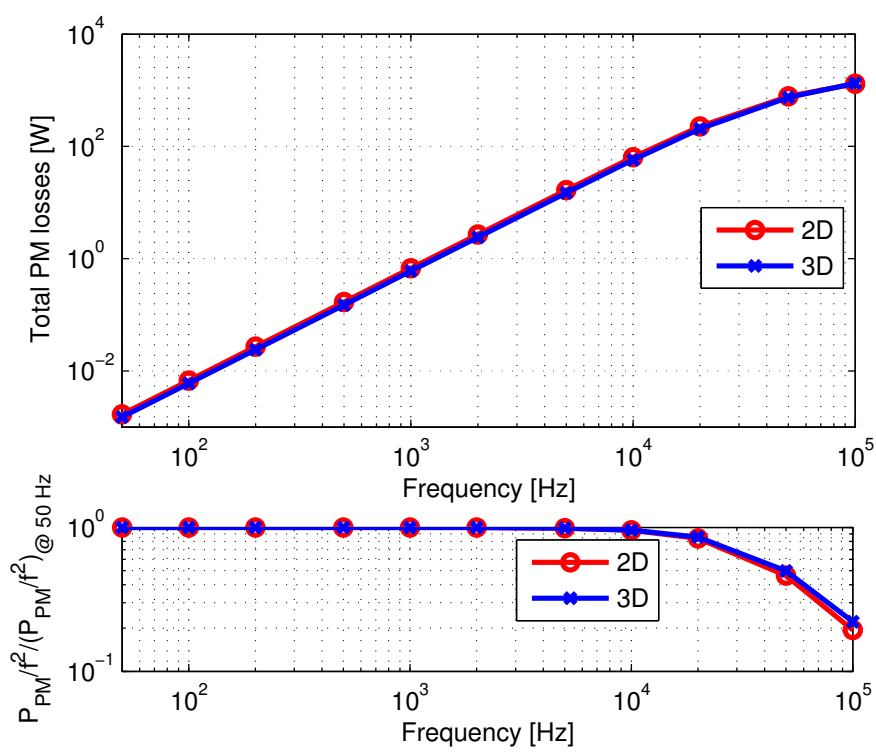

Fig. 3. Evolution of PM eddy-current losses with frequency: original values (top) and reduced values by considering the proportionality of losses with the frequency squared at sufficiently low frequency (below). The 2D and 3D reference meshes described in the next section are used for the computations.

is laminated [5].

\section{FE APPROACH TO COMPUTE EDDY CURRENTS}

The present model is supposed linearised and material is considered as isotropic (i.e. the reluctivity $\nu$ is assumed constant and scalar). The $b$-conform formulation of the 3D magnetic equations is chosen for the present calculations, i.e. with the use of the magnetic vector potential $\mathbf{A}=\operatorname{curl} \mathbf{B}, \mathbf{B}$ being the magnetic induction (or flux density):

$$
\operatorname{curl}(\nu \operatorname{curl} \mathbf{A})+\sigma \frac{\partial \mathbf{A}}{\partial t}-\mathbf{J}_{s}-\operatorname{curl}\left(\nu \mathbf{B}_{r}\right)=0
$$

with $\nu, \sigma, \mathbf{J}_{s}, t$ and $\mathbf{B}_{r}$ are the reluctivity and conductivity of the considered material, the imposed source current density, the time and the remanent induction of the PMs.

Ampère's law is then weakly imposed using weighing functions $\mathbf{w}_{j}$. By considering the complex (harmonic) formulation with the introduction of the pulsation $\omega=2 \pi f$ (with $f$ being the investigated frequency of stator currents):

$$
\int_{\Omega}\left(\operatorname{curl}(\nu \operatorname{curl} \mathbf{A})+j \omega \sigma \mathbf{A}-\mathbf{J}_{s}-\operatorname{curl}\left(\nu \mathbf{B}_{r}\right)\right) \mathbf{w}_{j} \mathrm{~d} \Omega=0 .
$$

The domain $\Omega$ is meshed and Whitney edge basis functions $\mathbf{w}_{i}$ are associated with the obtained edges to discretize the magnetic vector potential [8]. Those functions are the same as the weighing functions according to the Galerkin method:

$$
\mathbf{A}(x, y, z)=\sum_{i=1}^{N} a_{i} \mathbf{w}_{i}(x, y, z)
$$

where $a_{i}$ is the unknown associated with edge $i$ and $N$ the number of edges.

The discretized system can then be represented by the following (complex) matrix equation:

$$
\mathcal{S} \mathcal{A}+j \omega \mathcal{T} \mathcal{A}=\mathcal{J}
$$

where $\mathcal{A}$ is a column matrix grouping all $N$ unknowns, $\mathcal{S}$ the (square) stiffness matrix defined by:

$$
\mathcal{S}_{i, j}=\int_{\Omega} \nu \operatorname{curl}_{j} \cdot \operatorname{curl}_{i} \mathrm{~d} \Omega,
$$

$\mathcal{T}$ the (square) conductivity matrix defined (only inside the conducting domain $\Omega_{c}$ ) by:

$$
\mathcal{T}_{i, j}=\int_{\Omega_{c}} \sigma \mathbf{w}_{j} \cdot \mathbf{w}_{i} \mathrm{~d} \Omega_{c}
$$

and $\mathcal{J}$ the column matrix of the current densities:

$$
\mathcal{J}_{i}=\int_{\Omega} \mathbf{J} \cdot \mathbf{w}_{i} \mathrm{~d} \Omega
$$

As magnetic saturation is neglected, only fluctuating components of magnetic induction are relevant for the computation of eddy currents. As a consequence, the remanent flux of the PMs is neglected $\left(\mathbf{B}_{r}=0\right)$ in the investigated case. It has also to be noted that the paper focuses on the losses produced inside the PMs; the induced currents appearing in the stator and in the rest of the rotor are neglected (those parts are supposed to be made of sufficiently thin laminations to be considered as non-conductive)

Besides the Dirichlet boundary condition $(\mathbf{J} \cdot \mathbf{n}=0)$ on exterior border, anti-periodic conditions are imposed between the radial planes, as only one pole is modelled. A tree-cotree gauge condition is imposed for ensuring the unicity of $\mathbf{A}$. More details on the boundary and periodicity conditions can be found in [9]. 


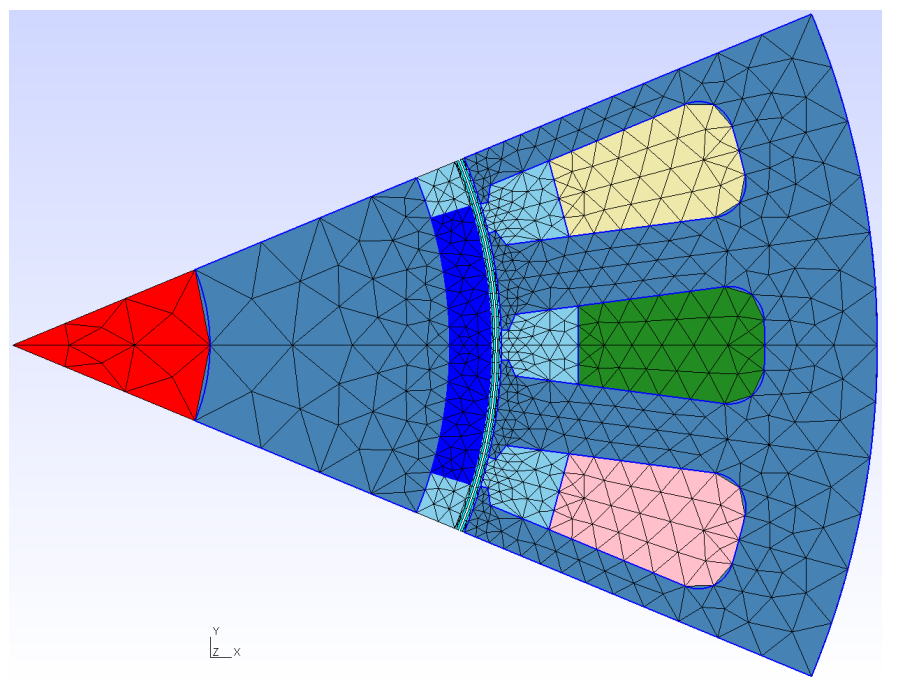

Fig. 4. Initial mesh used for the simulations with increasing $\delta$ and $\delta_{P M} . \delta$ and $\delta_{P M}$ are both equal to 1 .

\section{INFLUENCE OF DIVERSE FE PARAMETERS ON RESULTS}

All simulations are performed using the opensource ONELAB software (downloadable at http://onelab.info/wiki/ONELAB) [10], considering a $1 \mathrm{~A}$ amplitude $q$-axis current, that is, generating flux in quadrature with the magnet orientation ( $d$-axis). The cross-section of the PMSM is meshed with triangular elements; for 3D computations those triangles are extruded into prisms along the axial direction. The influence of FE mesh is investigated in three steps. First, 2D simulations are performed to investigate the effect on results of local and global mesh refinement in a large frequency range. The optimized 2D mesh got from step 1 is then extruded and 3D simulations are run to also investigate the influence of extrusion parameters on result accuracy. Finally, a comparison of $2 \mathrm{D}$ and $3 \mathrm{D}$ results is presented for different axial lengths. The computer on which all simulations are run comprises an Intel Core i3-3110M CPU $(2.4 \mathrm{GHz}), 8 \mathrm{~GB}$ of RAM and a 64-bit Windows 10 operating system.

\section{A. Influence of $2 D$ relative mesh density}

The influence of the global and local mesh density is investigated in this subsection, by performing simulations starting from the coarse mesh shown in Figure 4. This mesh is used as a reference for the definition of the global and local mesh refinement coefficients $\delta$ and $\delta_{P M}$ (both equal to 1 in that case). Those coefficients are gradually increased up to $\delta$ and $\delta_{P M}$ equal to 6 and 20 respectively, while considering various combinations at $100 \mathrm{~Hz}$ and at $100 \mathrm{kHz}$. The effect of doubling one or the other refinement coefficient on the mesh is illustrated in Figures 5 and 6. As it can be observed on the figures, doubling $\delta$ doubles the number of nodes on each line of the model, while doubling $\delta_{P M}$ has only this effect at the location of the magnets, while the mesh remains unchanged far from them.

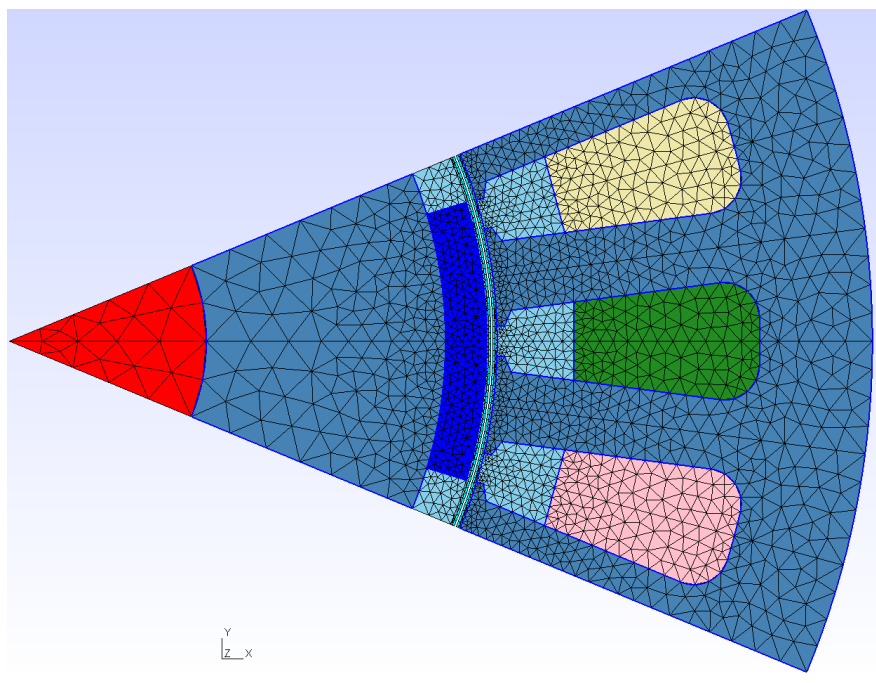

Fig. 5. Obtained mesh with $\delta$ and $\delta_{P M}$ equal to 2 and 1 respectively.

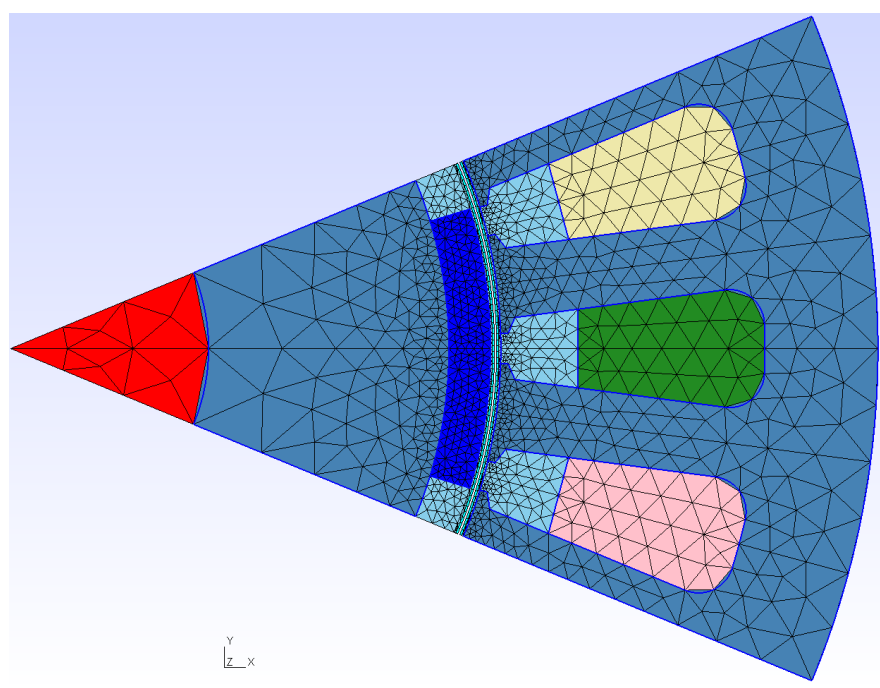

Fig. 6. Obtained mesh with $\delta$ and $\delta_{P M}$ equal to 1 and 2 respectively.

The simulation case with the finest mesh $(\delta=6$ and $\delta_{P M}=20$, as shown in Figure 7) is chosen as a reference to compute eddy-current losses. The error on computed losses is plotted in function of simulation time at $100 \mathrm{~Hz}$ and at 100 $\mathrm{kHz}$ in Figures 8 and 9 respectively. On these plots each curve corresponds to a constant value of $\delta_{P M}$, while $\delta$ increases from 1 to 6 by step of 1 from left to right.

The results shown on the plot roughly follow a trend line showing that the error can be reduced by a factor $n$ at the cost of an increase in computation time by approximately $n^{2}$. It also turns out that the simulations with $\delta_{P M}=5$ generally give the lowest errors compared to other values of $\delta_{P M}$ for the same computation time. This conclusion can be drawn at both low and high frequencies. Therefore, this value of $\delta_{P M}$ is kept constant and equal to 5 for the rest of the simulations. 


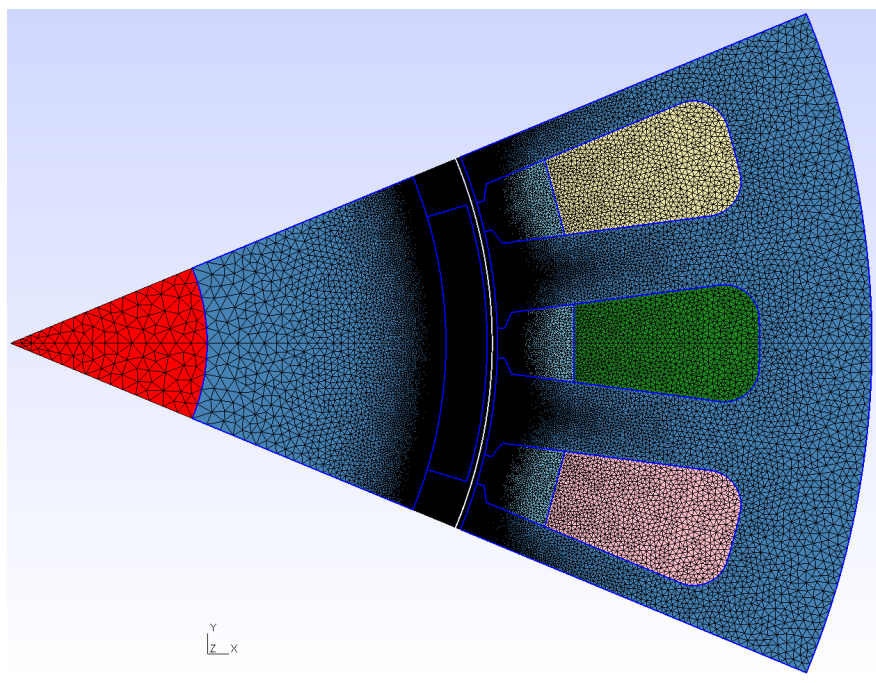

Fig. 7. Finest mesh used as a reference for result comparison to get the optimal value of $\delta_{P M} . \delta$ and $\delta_{P M}$ are equal to 6 and 20 respectively.

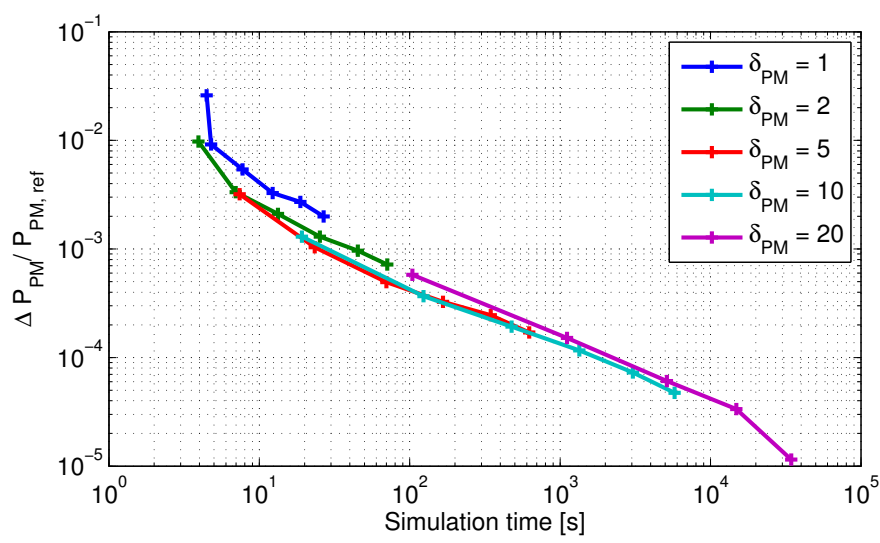

Fig. 8. Evolution of the error on eddy-current losses and of computation time with $\delta$ equal to 1 to 6 (from left to right) at $100 \mathrm{~Hz}$ for different values of $\delta_{P M}$. The losses computed for $\delta=6$ and $\delta_{P M}=20$ are chosen as the reference.

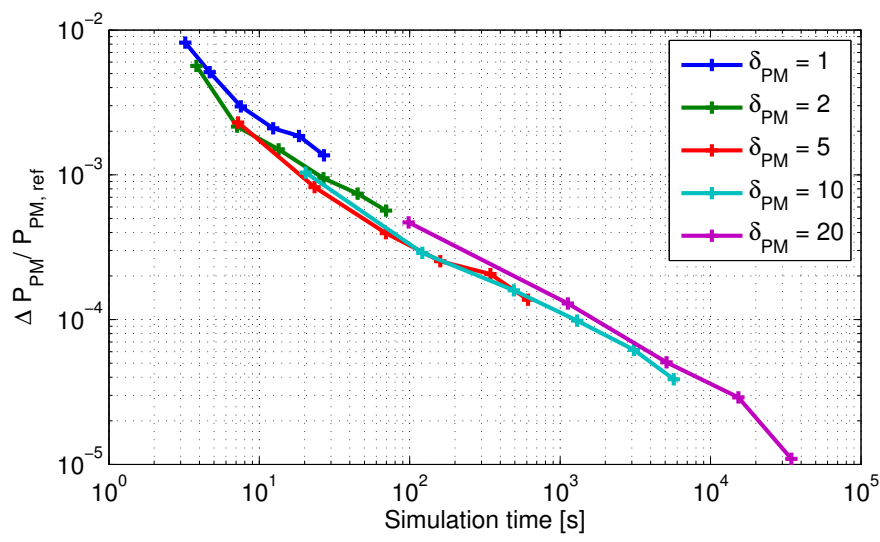

Fig. 9. Evolution of the error on eddy-current losses and of computation time with $\delta$ equal to 1 to 6 (from left to right) at $100 \mathrm{kHz}$ for different values of $\delta_{P M}$. The losses computed for $\delta=6$ and $\delta_{P M}=20$ are chosen as the reference.
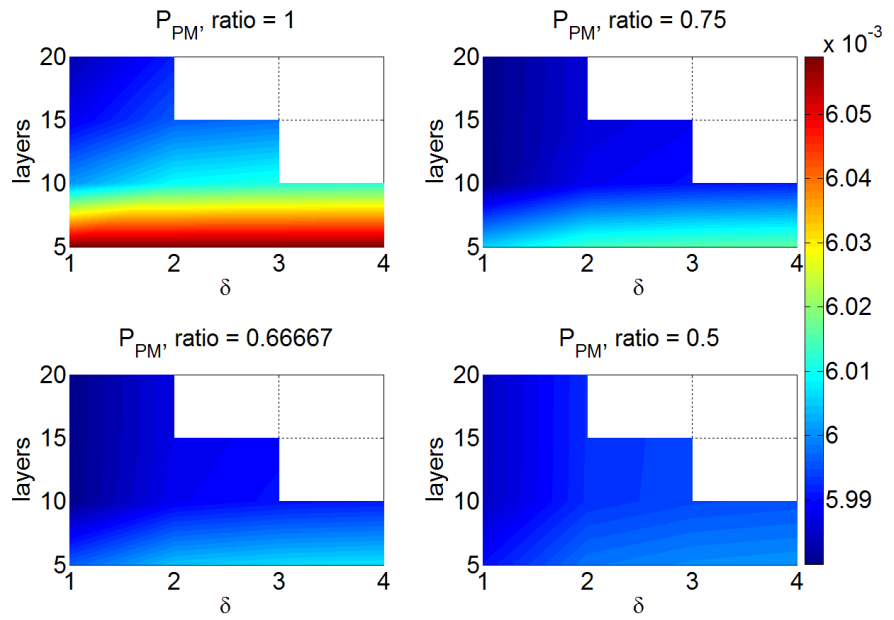

Fig. 10. Evolution of the computed eddy-current losses $P_{P M}$ in W when varying $\delta, n_{l, z}$ and $\rho_{l, z}$ at $100 \mathrm{~Hz}$.
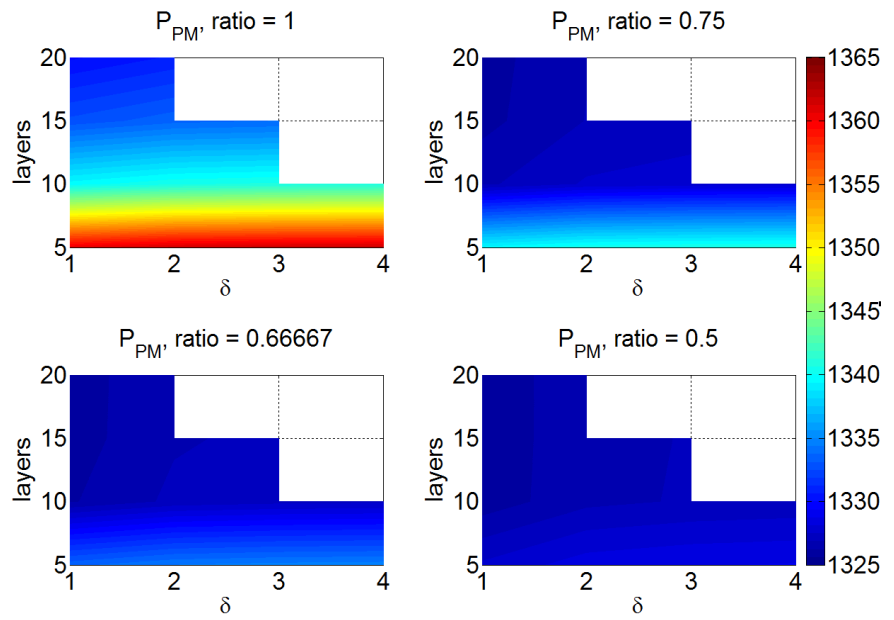

Fig. 11. Evolution of the computed eddy-current losses $P_{P M}$ in When varying $\delta, n_{l, z}$ and $\rho_{l, z}$ at $100 \mathrm{kHz}$.

\section{B. Influence of number and distribution of axial layers}

In order to investigate the influence of the number of axial layers $n_{l, z}$ and of the thickness ratio between two consecutive layers $\rho_{l, z}$ together with the one of the mesh refinement factor $\delta$ on the results, 3D FE calculations are performed for all combinations of parameters, considering $\delta=\{1,2,3,4\}$, $n_{l, z}=\{5,10,15,20\}$ and $\rho_{l, z}=\{1 / 2,2 / 3,3 / 4,1\}$. The computed value of eddy-current losses in the PMs is plotted as colour maps for $100 \mathrm{~Hz}$ and $100 \mathrm{kHz} q$-axis currents in Figures 10 and 11 . Due to memory limitation, no results could be obtained for some of the combinations, leading to the absence of the upper-right part of the maps.

As expected using axial layers with identical width gives the least accurate results. For the three other values of $\rho_{l, z}$ the result is nearly independent from $\delta$ when less than 10 layers are used, while it is nearly independent from the number of layers if $\delta<2$. These two observations suggest that using values of $\delta$ and of $n_{l, z}$ smaller than 2 and 10 respectively are not appropriate if accurate computations are needed. The 


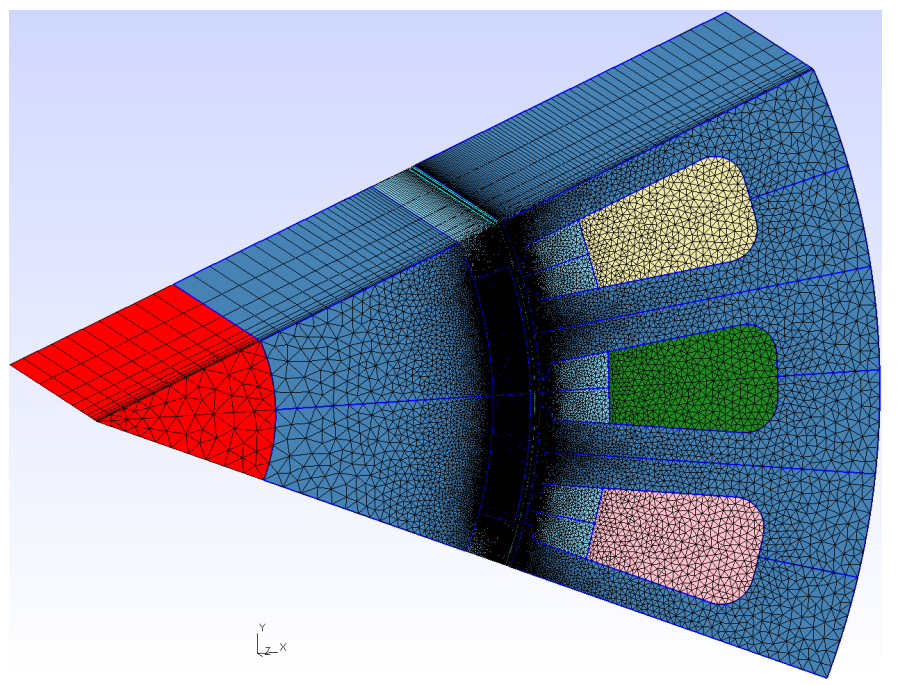

Fig. 12. Used geometry and mesh for the reference case. Only one pole over half the axial length of the PMSM is represented. $\delta, \delta_{P M}, n_{l, z}$ and $\rho_{l, z}$ are set to $4,5,10$ and $2 / 3$ respectively.

results for values of $\delta$ and of $n_{l, z}$ between 2 and 3 and between 10 and 15 respectively are similar for $\rho_{l, z}$ equal to $2 / 3$ and $3 / 4$. They are also similar to the case of $\rho_{l, z}=1 / 2$, but only at $100 \mathrm{kHz}$. The explanation is linked to the trend of the current density to concentrate near the surface of the conductors as the frequency increases; a smaller progression factor is then better suited at high frequency to model the U-turns of eddy currents with more layers.

Therefore, as there is a need of choosing a basis for the comparison of $2 \mathrm{D}$ and $3 \mathrm{D}$ computations in the next subsection, $\rho_{l, z}=2 / 3$ can be selected as the best trade-off for both cases. The selection of $\delta$ and of $n_{l, z}$ for the reference calculation are performed by observing the influence of both parameters in the range of $2<\delta<3$ and $10<n_{l, z}<15$. As the colour gradient appears to be more horizontal in that zone, $\delta$ is considered to have more influence. As a consequence, the following parameters are fixed for the reference mesh: $\delta=4$, $\delta_{P M}=5, n_{l, z}=10$ and $\rho_{l, z}=2 / 3$. The used mesh for the $3 \mathrm{D}$ reference case is shown in Figure 12. It has to be noted that the $2 \mathrm{D}$ reference mesh also used hereafter corresponds to the cross-section of the $3 \mathrm{D}$ reference one.

Results obtained with the $2 \mathrm{D}$ and $3 \mathrm{D}$ reference meshes in terms of flux lines and of eddy-current density are presented in Figures 13 to 20 for a $q$-axis current pulsating at $100 \mathrm{~Hz}$ and $100 \mathrm{kHz}$. As the apparition of eddy currents leads to a phase shift between the imposed $q$-axis current and the flux, results are presented as real and imaginary components, that is, components in phase and in quadrature with the imposed winding current. It has also to be noted that the eddy currents are nearly in quadrature with the winding currents at low frequency, leading to the use of different scales for real and imaginary parts.

As it can be observed in Figures 13 to 16, the real and imaginary parts of the flux are essentially generated by the $q$-axis winding current and by the eddy currents respectively.

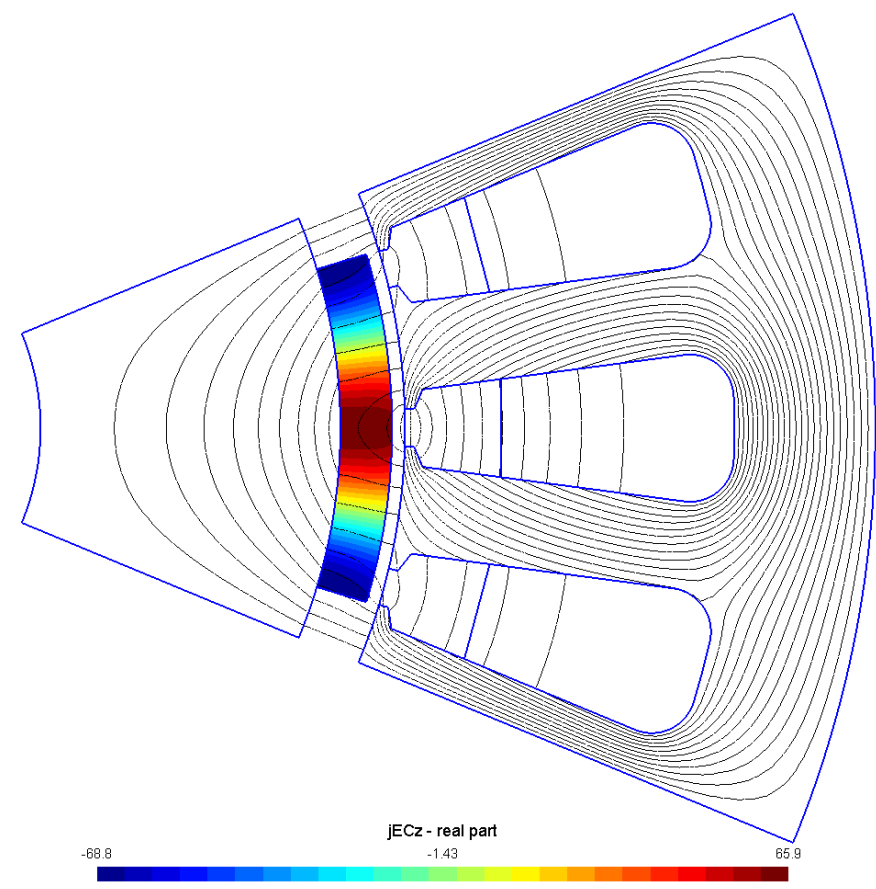

Fig. 13. Flux lines and induced-current density plot obtained for a $1 \mathrm{~A} 100 \mathrm{~Hz}$ $q$-axis current in the 2D FE reference case. The minimum and maximum of the $\mathrm{z}$ component of the magnetic vector potential are $-6.57 \times 10^{-4}$ and $1.15 \times 10^{-11}$ respectively.

The shielding effect of the induced currents is also visible at $100 \mathrm{kHz}$ in Figure 15, while the concentration of the currents in the periphery of the PM is also visible at that frequency in Figure 16. This phenomenon is also illustrated in Figures 17 to 20 by 3D FE computations. The current density vectors are displayed on a cylindrical surface cutting the PM at middepth. The left and right sides of the figures correspond to the middle and end transversal planes of the machine respectively.

\section{Comparison of $2 D$ and $3 D$ results and influence of axial length}

The error linked with the use of $2 \mathrm{D}$ instead of $3 \mathrm{D} F E$ is investigated here by comparing the PM losses computed in $2 \mathrm{D}$ and in $3 \mathrm{D}$ using the here-above defined reference meshes (having the same cross-section characteristics). It has to be noted that the integral of the axial current inside the PMs is explicitly imposed to be zero in 2D as it is implicitly in 3D. The absolute differences between PM losses computed with $3 \mathrm{D}$ and 2D FE are presented at four frequencies $(100 \mathrm{~Hz}$, $1 \mathrm{kHz}, 10 \mathrm{kHz}$ and $100 \mathrm{kHz}$ ) for different stack lengths $L_{z}$ from $4 \mathrm{~cm}$ to $1 \mathrm{~m}$ in the upper part of Figure 21. For better clarity of this figure (and of the next ones) logarithmic scales are used for both axes. Black and red markers are used when the relative differences are positive and negative respectively. As expected the 2D model is more and more valid as the value of stack length increases (the error falls below $5 \%$ for stack lengths of $10 \mathrm{~cm}$ and above).

Relying on analytical approaches, the 2D case may be corrected to consider end effects by multiplying the $2 \mathrm{D}$ result 


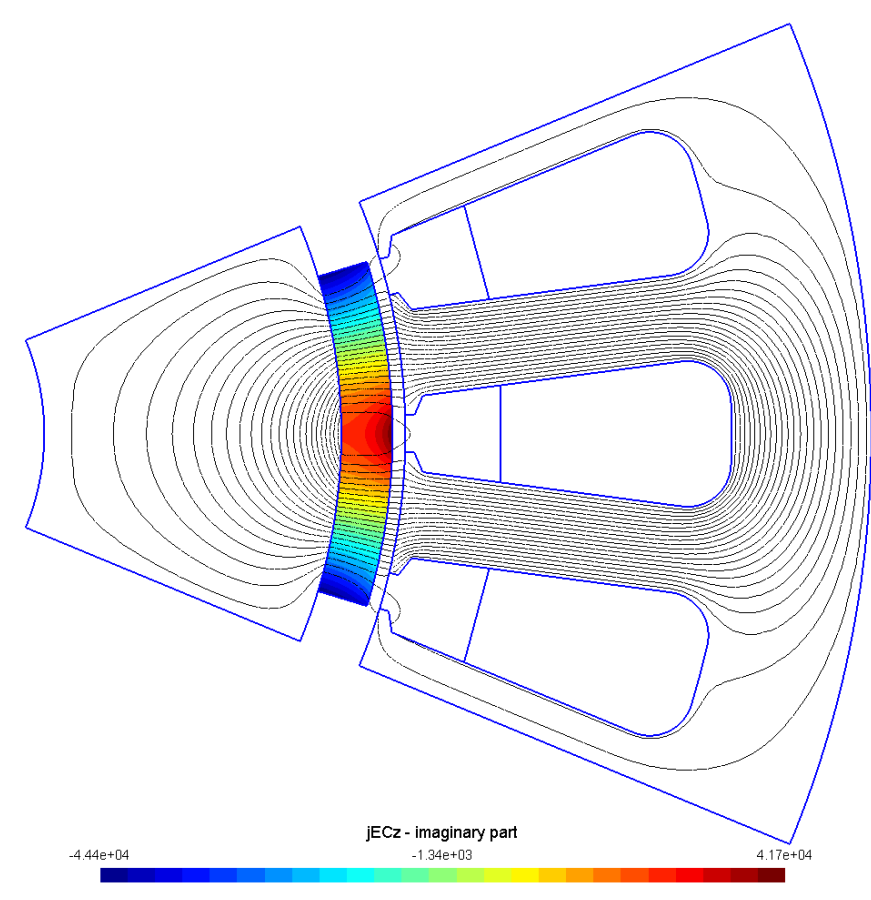

Fig. 14. Flux lines and induced-current density plot obtained for a $1 \mathrm{~A} 100 \mathrm{~Hz}$ $q$-axis current in the $2 \mathrm{D} \mathrm{FE}$ reference case. The minimum and maximum of the $\mathrm{z}$ component of the magnetic vector potential are $-5.57 \times 10^{-9}$ and $3.14 \times 10^{-7}$ respectively.

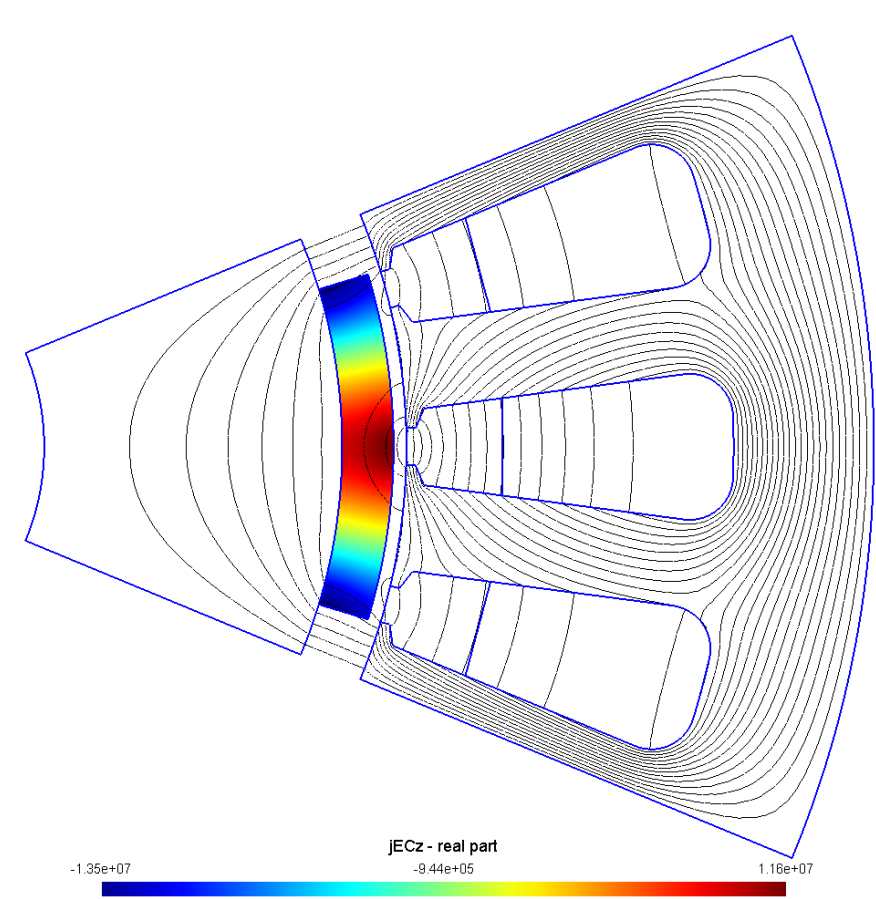

Fig. 15. Flux lines and induced-current density plot obtained for a 1 A $100 \mathrm{kHz} q$-axis current in the 2D FE reference case. The minimum and maximum of the $\mathrm{z}$ component of the magnetic vector potential are $-5.47 \times 10^{-4}$ and $8.05 \times 10^{-12}$ respectively.

with a slightly modified version of the correction coefficient

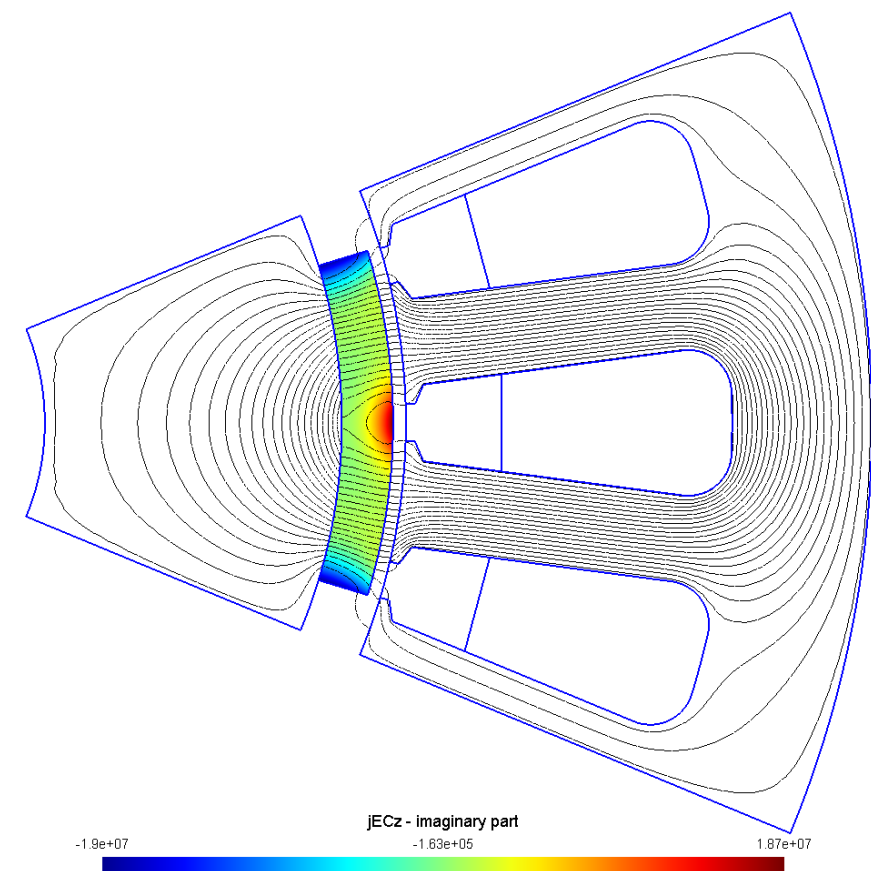

Fig. 16. Flux lines and induced-current density plot obtained for a $1 \mathrm{~A}$ $100 \mathrm{kHz} q$-axis current in the $2 \mathrm{D}$ FE reference case. The minimum and maximum of the $\mathrm{z}$ component of the magnetic vector potential are $-1.95 \times 10^{-6}$ and $5.78 \times 10^{-5}$ respectively.

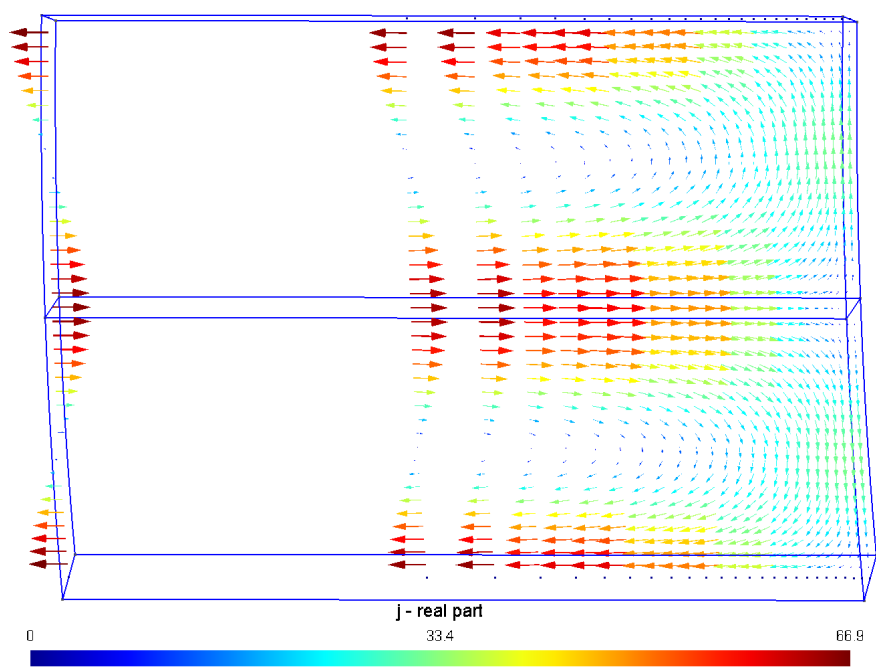

Fig. 17. 3D vector map of the induced currents in half of the PM obtained for a $1 \mathrm{~A} 100 \mathrm{~Hz} q$-axis current in the 3D FE reference case.

$k_{L}$ used in [2]:

$$
k_{L}=\frac{L_{z}^{2}}{L_{z}^{2}+l_{P M}^{2}}
$$

with $l_{P M}$ the width of the PM. The modified ratios obtained by multiplying the $2 \mathrm{D}$ result with that coefficient show a better agreement between $2 \mathrm{D}$ and $3 \mathrm{D}$ (the error remains below $5 \%$ even for $4 \mathrm{~cm}$ stack length, except at $10 \mathrm{kHz}$ ), even if some overcompensation of the error can be remarked as the error change signs for the lowest stack length (black marks). 


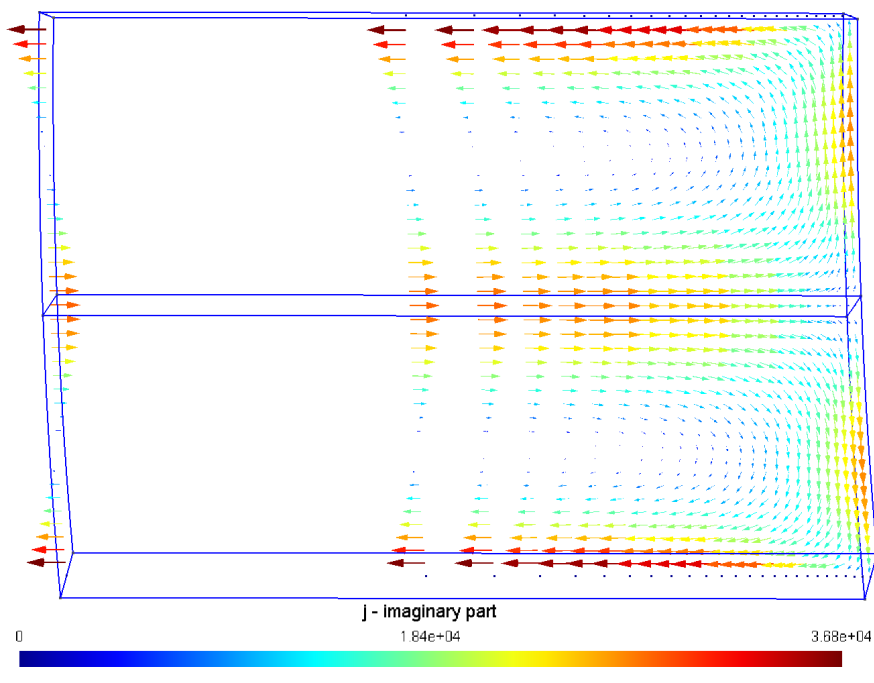

Fig. 18. 3D vector map of the induced currents in half of the PM obtained for a $1 \mathrm{~A} 100 \mathrm{~Hz} q$-axis current in the 3D FE reference case.

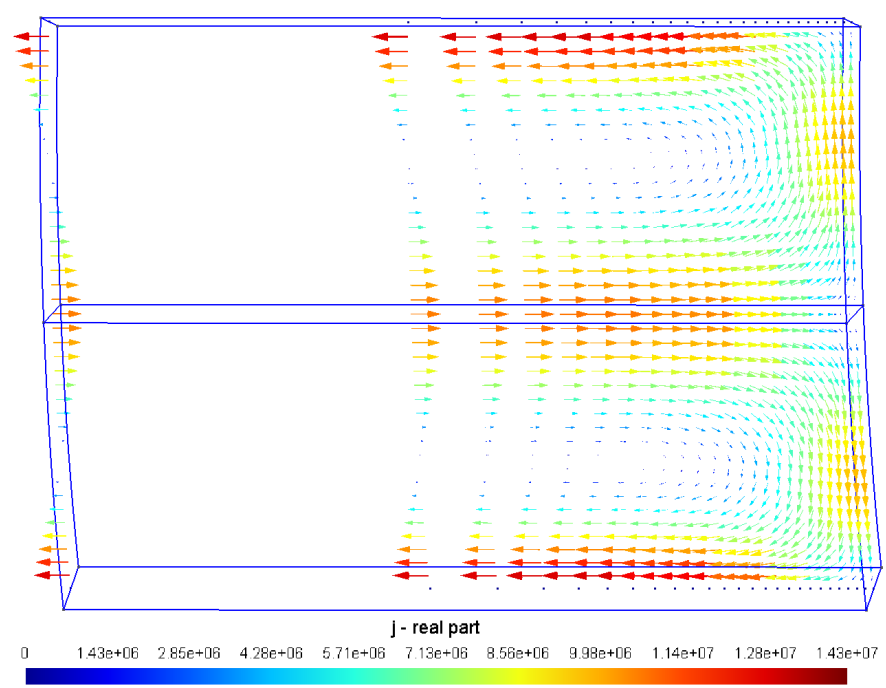

Fig. 19. 3D vector map of the induced currents in half of the PM obtained for a $1 \mathrm{~A} 100 \mathrm{kHz} q$-axis current in the 3D FE reference case.

\section{CONCLUSION}

In this paper the evolution of results with several mesh parameters when calculating the eddy-current losses in a PMSM have been presented. It turns out that globally increasing the mesh density appears as the most effective technique to gain accuracy. Increasing the $2 \mathrm{D}$ mesh density or adding axial layers increases result accuracy, but at the expense of a higher computation time. Considering the nature of modelled phenomena and the geometry of the machine, trade-offs can be made to obtain a better ratio between sustainable computation costs and result accuracy. Moreover, attention must be given to all parameters, as the influence of one parameter on the accuracy may become negligible if another parameter is wrongly set. However, the better performance of one value compared to others cannot be concluded. 2D simulations may be an interesting low-cost alternative to $3 \mathrm{D} \mathrm{FE}$, as far as

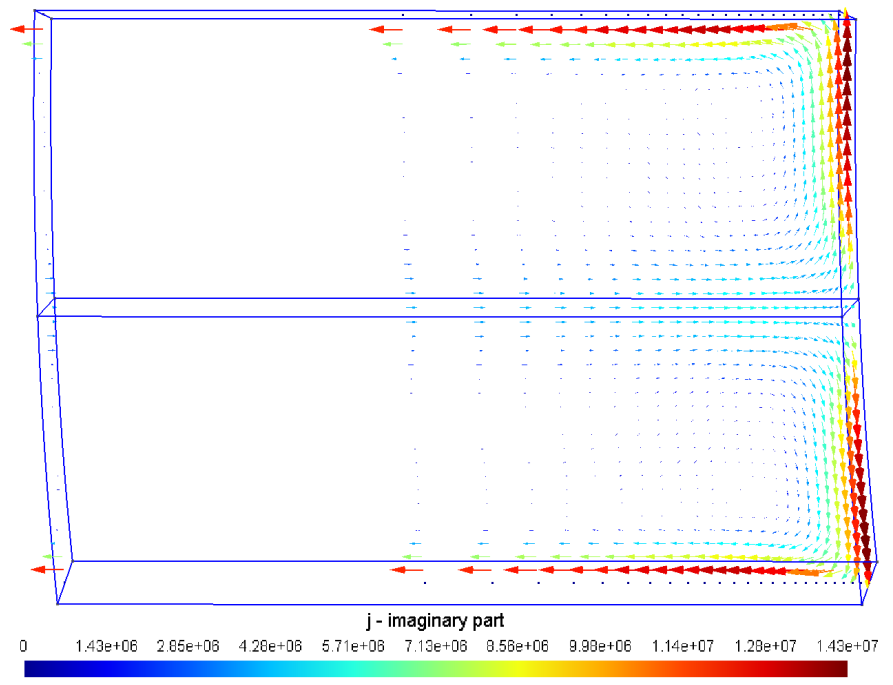

Fig. 20. 3D vector map of the induced currents in half of the PM obtained for a 1 A $100 \mathrm{kHz} q$-axis current in the 3D FE reference case.
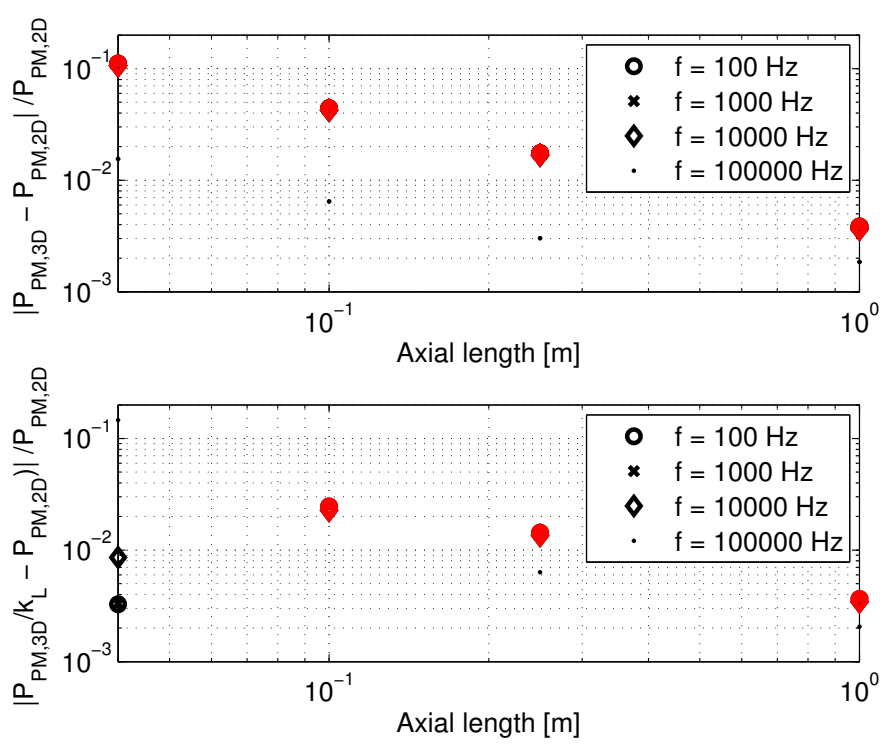

Fig. 21. Evolution with frequency of the relative differences of PM losses computed with $3 \mathrm{D}$ and $2 \mathrm{D} \mathrm{FE}$. The correction factor $k_{L}$ is taken into account in the lower plot. $\delta, \delta_{P M}, n_{l, z}$ and $\rho_{l, z}$ are $2.5,2,20$ and $2 / 3$ respectively; black and red markers are used for positive and negative of the relative differences respectively.

the stack length is sufficiently high. When the end-effect correction factor is taken into account, relative good results can be obtained with the present geometry, even for comparable values of external diameter and stack length for highly reduced computational costs.

Future work will include the influence of the rotor position in the present study. The use of a finer mesh for the reference test case may be used, but needs the usage of a more powerful (non-standard) computer. The influence of parameters can then be investigated referring to a finer mesh, to observe in which extent a reduction of computational cost does not increase too much the error. 


\section{ACKNOWLEDGMENT}

The authors would like to thank the Walloon Region for financing this research through the FEDO project.

\section{REFERENCES}

[1] S. Jumayev and M. Merdzan and K.O. Boynov and J.J.H. Paulides and J. Pyrhonen and E. A. Lomonova, "The Effect of PWM on Rotor EddyCurrent Losses in High-Speed Permanent Magnet Machines," in IEEE Transactions on Magnetics, vol. 51, no. 11, 2015.

[2] F. Martin and M.E.H. Zaim and A. Tounzi and N. Bernard, "Improved analytical determination of eddy current losses in surface mounted permanent magnets of synchronous machine," in IEEE Transactions on Magnetics, vol. 50, no. 6, pp. 19, 2014.

[3] R. Fratila and A. Benabou and A. Tounzi and J.-C. Mipo, "A combined experimental and finite element analysis method for the estimation of eddy-current loss in NdFeB magnets," in Sensors, vol. 14, no. 5 , 85058512, 2014.

[4] M. Mirzaei and A. Binder and B. Funieru and M. Susic,"Analytical calculations of induced eddy currents losses in the magnets of surface mounted PM machines with consideration of circumferential and axial segmentation effects," in IEEE Transactions on Magnetics, vol. 48, no. 12, pp. 48314841, 2012.

[5] P. Sergeant and A. Van Den Bossche, "Segmentation of magnets to reduce losses in permanent-magnet synchronous machines," in IEEE Transactions on Magnetics, vol. 44, no. 11, 2008.

[6] S. Steentjes and S. Boehmer and K. Hameyer, "Permanent magnet eddycurrent losses in 2-D FEM simulations of electrical machines," in IEEE Transactions on Magnetics, vol. 51, no. 3, pp. 36, 2015.

[7] J. Gyselinck and N. Sadowski and P. Dular and M.V. Ferreira da Luz and J.P.A. Bastos and W. Legros, W., "Harmonic Balance Finite Element Modelling of a Permanent-Magnet Synchronous Machine," in Proceedings of the V Brazilian Conference on Electromagnetics, Gramado, Brazil, pp. 14, 2002

[8] A. Bossavit, "Whitney forms: a class of finite elements for threedimensional computations in electromagnetism," in IEE Proceedings, vol. 135, pt. A, no. 8, pp. 493-500, 1988.

[9] M.F. Da Luz and P. Dular and N. Sadowski and C. Geuzaine and J.P.A Bastos, "Analysis of a permanent magnet generator with dual formulations using periodicity conditions and moving band," in IEEE Transactions on Magnetics, vol. 38, no. 2, pp. 961-964, 2002.

[10] Johan J. C. Gyselinck and Adrian Cornel Pop, "Finite-Element and Lumped Parameter Modelling and Simulation of Permanent Magnet Synchronous Machines - From Academic State of the Art to Design Office Practice: Tutorial presented at the XXIIth International Conference on Electrical Machines (ICEM'2016)," 2016. 